\title{
Incidence of and Effects of Seasonality on Non-purulent Lower Extremity Cellulitis after the Emergence of Community-acquired Methicillin-resistant Staphylococcus aureus
}

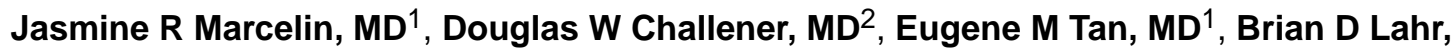 \\ MS $^{3}$, and Larry M Baddour, MD' \\ ${ }^{1}$ Division of Infectious Diseases, Department of Internal Medicine, College of Medicine, Mayo \\ Clinic, 200 1st St SW, Rochester, Minnesota \\ 2Department of Internal Medicine, College of Medicine, Mayo Clinic, 2001 st St SW, Rochester, \\ Minnesota \\ ${ }^{3}$ Division of Biomedical Statistics and Informatics, Department of Health Sciences Research, \\ College of Medicine, Mayo Clinic, 200 1st St SW, Rochester, Minnesota
}

\begin{abstract}
Non-purulent lower extremity cellulitis (NLEC) is a common clinical diagnosis with betahemolytic streptococci and Staphylococcus aureus considered as the most frequent causes. In 1999, the United States Public Health Service alerted clinicians of community-acquired methicillin-resistant Staphylococcus aureus (CA-MRSA) infections in four children in the Upper Midwest. Since then, it has become a well-recognized cause of skin and soft tissue infections, in particular, skin abscess. A previous population-based study of NLEC in Olmsted County, Minnesota reported an unadjusted incidence rate of 199 per 100,000 person-years in 1999, but it is unknown whether CA-MRSA subsequently has impacted NLEC incidence. We therefore sought to determine the population-based incidence of NLEC since the emergence of CA-MRSA. Age- and sex-adjusted incidence (per 100,000 persons) of NLEC was 176.6 (95\% CI: 151.5, 201.7). Incidence differed significantly between sexes with age-adjusted sex-specific rates of 133.3 (95\% CI: 104.1, 162.5) and 225.8 (95\% CI: 183.5, 268.0) in females and males, respectively. Seasonal incidence differed, with rates of 224.6 (95\% CI: 180.9, 268.4) in warmer months (MaySeptember) as compared to 142.3 (95\% CI: $112.8,171.9)$ in colder months (January-April and October-December). Despite emergence and nationwide spread of CAMRSA since 1999 in the United States, the incidence of NLEC in Olmsted County was lower in 2013 than in 1999, particularly among females. This suggests that CA-MRSA is not a significant cause of NLEC and
\end{abstract}

Corresponding author: Jasmine R Marcelin MD, Division of Infectious Diseases, Mayo Clinic, 200 1st St SW Rochester, MN 55905. marcelin.jasmine@mayo.edu. Phone: 507-284-3309. Fax: 507-255-7767.

Conflicts of interest: JRM, DWC, EMT, BDL, LMB - No conflicts of interest for any authors

*Portions of the data were presented at IDWeek 2016 ${ }^{\mathrm{TM}}$ (October 28, 2016); abstract selected for IDWeek $2016^{\mathrm{TM}}$ Trainee Travel Grant

Publisher's Disclaimer: This is a PDF file of an unedited manuscript that has been accepted for publication. As a service to our customers we are providing this early version of the manuscript. The manuscript will undergo copyediting, typesetting, and review of the resulting proof before it is published in its final citable form. Please note that during the production process errors may be discovered which could affect the content, and all legal disclaimers that apply to the journal pertain. 
that NLEC cases are seasonally distributed. These findings may be important in formulation of empiric therapy for NLEC and in patient education as many patients with NLEC are prone to recurrent bouts of this infection.

\section{Keywords}

cellulitis; seasonality; incidence; population-based; community-acquired methicillin-resistant Staphylococcus aureus

\section{Introduction}

Non-purulent lower extremity cellulitis (NLEC) is one of the most common skin/soft tissue infections (SSTI) encountered by clinicians. ${ }^{1,2}$ Despite its frequency in clinical practice, few studies describe the incidence of NLEC, which has ranged from 19 to 2740 per 100,000 person-years. ${ }^{3-6}$ Most of these studies did not include individual medical record review, and by using only ICD-9 codes, their incidence figures included patients with purulent SSTIs, because cellulitis and skin abscess share a common ICD-9 code (681.x). To our knowledge, only one study has described the incidence of NLEC in a population-based cohort in the United States (199 per 100,000 person-years in 1999) and included individuals from Olmsted County, Minnesota. ${ }^{7}$

Community-acquired methicillin-resistant Staphylococcus aureus (CA-MRSA) has been described as early as the 1980s as a cause of bacterial endocarditis in intravenous drug abusers. $^{8}$ The first cases of CA-MRSA causing purulent SSTIs were seen in the 1990s in the USA. ${ }^{9}, 10$ In response to an increase in infections due to CA-MRSA, the United States Public Health Service began monitoring the prevalence of CA-MRSA infections in this country. Changes in the prevalence of CA-MRSA in Minnesota have been prospectively monitored by 12 sentinel hospital-based laboratories throughout the state. ${ }^{11}$ In 2000 , the prevalence of CAMRSA infections among all MRSA infections in Minnesota was 11\% and had significantly increased to $33 \%$ by the end of $2005 .{ }^{12}$ The majority of CA-MRSA infections were SSTIs, increasing from $75 \%$ to $87 \%$ between 2000 and $2005 .{ }^{12}$

Whether CA-MRSA has caused a similar increase in NLEC cases, however, remained undefined. Establishing or refuting a causal link between CA-MRSA and NLEC is difficult because microbiologic data are not obtained in the bulk of NLEC cases. Due to the overall increase in SSTI prevalence since the emergence of CA-MRSA, there may be an understandable inference that CA-MRSA may play a role in NLEC. Such inferences may lead to empiric antimicrobial prescribing practices that include unnecessary coverage for CA-MRSA. Therefore, if one could demonstrate that despite the increase in incidence of SSTIs caused by CA-MRSA, the incidence of NLEC had remained unchanged, then this could justify the selection of empiric antibiotic therapy for NLEC that does not include coverage for CA-MRSA. In this regard, no studies have evaluated the influence of CAMRSA on the incidence of NLEC. In the present study, we therefore define the incidence of NLEC after the emergence of CA-MRSA in the US by using the same population-based cohort evaluated by our group in $1999 .{ }^{7}$ 


\section{Methods}

This retrospective, population-based study was conducted in Olmsted County, Minnesota, among adult residents with NLEC between January and December 2013. Medical records were accessible through the Rochester Epidemiology Project (REP), a medical record linkage system for all county-based medical facilities. ${ }^{13}$ The initial set of potential cases was identified using the same ICD-9 codes for cellulitis and abscess as were used in the 1999 study $^{7}$ (Supplementary Table 1). Current Procedural Terminology (CPT) codes were applied to exclude patients with purulent SSTIs requiring procedural drainage (Supplementary Table 2).

Medical records were reviewed and cases were initially included or excluded by trained nurse abstractors. JRM re-reviewed cases to confirm that they met the case definition or were appropriately excluded. LMB independently randomly reviewed 5\% (93) of all charts; agreement was $96 \%$ on chart classification. Without laboratory "gold standards" for NLEC diagnosis, previous studies have accepted the evaluating clinician's diagnosis. Our case definition is consistent with that used in recent studies. ${ }^{7,14,15}$ NLEC was defined as a clinician's clinical diagnosis with documentation of acute onset of warmth, erythema, and edema on the lower extremities. Excluded patients had purulent or necrotizing SSTIs, osteomyelitis, secondary skin infections, burn wounds, infected prosthetic material or infected human or animal bite wounds. Warmer months (May-September) and colder months (January-April and October-December) were predetermined according to seasonal periods identified in the 1999 study. $^{7}$

\section{Statistical analysis}

To examine the incidence of NLEC, count data for cases and total person-time were generated for subsets of the local population defined by sex and age (in single years from age 18 to 100). Numbers of persons in Olmsted County were ascertained via linkage with data sources from REP, which provide a reliable means for enumerating population estimates. ${ }^{16}$ Unadjusted incidence rates were derived using counts of cases in the numerator and the corresponding counts of the county population in the denominator. Age- and sexadjusted rates were computed based on direct standardization against the 2010 U.S. White population, ${ }^{17}$ with $95 \%$ confidence intervals estimated using the Poisson distribution.

All incidence rates are presented per 100,000 person-years; calculations of monthly and seasonal incidence rates required correcting the population counts to compensate for an observation period < 1 year (e.g., for monthly incidence, counts were multiplied by a constant of 1/12). Smoothing techniques based on the non-parametric loess method were used to illustrate the relation of age, sex and calendar time with incidence rate, and multivariable Poisson regression was used to test for independent differences in rates between the levels of each risk factor. The model was formulated with number of cases as the dependent variable and number of total person-years on log scale as an offset variable; independent covariates included age as a continuous variable, and sex and season (warm/ cold) as binary variables. Analyses were performed with SAS statistical software (Version 9.4, SAS Institute Inc., Cary, NC). Further details of the statistical rationale associated with incidence comparisons are outlined in Supplemental Box 1. 


\section{Human Subject Protection}

The Mayo Clinic and Olmsted County Medical Center Institutional Review Boards approved this study. All patients participating in the REP have previously consented to the research use of their medical records.

\section{Results}

\section{Cohort Characteristics}

1867 records were initially identified from REP as potential NLEC episodes based on diagnostic codes. Of these, 195 (10.4\%) individuals met our definition for incident cases of NLEC. Most patients were Caucasian (96.4\%), males (57.9\%), and were diagnosed in the outpatient setting (67.2\%). All patients presented with erythema and received antibiotics. Twenty-eight patients (14.4\%) required hospitalization for a median duration of 3.5 days (Table 1).

\section{Incidence}

During 2013, the unadjusted incidence rate of NLEC was 168.6 per 100,000 persons. When standardized to the 2010 US White population, the overall age- and sex-adjusted incidence (per 100,000 persons) of NLEC was 176.6 (95\% CI: 151.5, 201.7), with age-adjusted rates of 133.3 (95\% CI: 104.1, 162.5) in females and 225.8 (95\% CI: 183.5, 268.0) in males (Table 2). Multivariable Poisson regression analysis revealed that incidence of NLEC was significantly higher in males than females $(\mathrm{P}<.001)$ and that the incidence rates increased with older age $(P<.001)$; Figure 1$)$.

\section{Seasonal Variation}

Table 2 and Supplementary Figure 1 show the seasonal effect on incidence of NLEC, with age-and sex-adjusted rates (per 100,000 persons) of 224.6 (95\% CI: 180.9-268.4) in the warmer months (May-September) compared to 142.3 (95\% CI: 112.8-171.9) in the colder months (January-April, October-December). In the multivariable model adjusted for age and sex, this seasonal difference was statistically significant $(\mathrm{P}=.001)$ and corresponded to a 1.6 (95\% CI: 1.2-2.1) -fold higher incidence in warmer months. From sex-stratified models, the seasonal difference was significant in females (2.2-fold higher in warmer months; $\mathrm{P}<$. 001) and non-significant in males (1.3-fold higher in warmer months; $\mathrm{P}=.19$ ).

\section{Discussion}

A comparison of the incidence of NLEC before and after the emergence of CA-MRSA is of keen interest since it is widely recognized as a cause of SSTI, predominately skin abscess. Our investigation was performed in the same setting with the same case definition as a previous study of the incidence of NLEC in Olmsted County, Minnesota in 1999, which found an unadjusted incidence of 199.2 per 100,000 persons. ${ }^{7}$ However, when contrasting the incidence rates between the current and previous study, it is important to account for potential age and sex differences in the local population between 1999 and 2013. To improve the comparability between studies, the 1999 incidence was re-evaluated by standardizing rates to the age and sex distributions of the base population (2010 US Whites) used in the 
current study (described in detail in the supplemental materials). In addition, aggregate data from 1999 and 2013 was analyzed with statistical modeling to compensate for population differences, allowing a direct comparison of rates between years to investigate changes in incidence of NLEC since CA-MRSA emergence. The overall age- and sex-adjusted incidence of NLEC for 1999 was 216.0 per 100,000, exceeding the corresponding rate of 176.6 per 100,000 that we report for $2013(\mathrm{P}=.045)$. This suggests that the incidence of NLEC in Olmsted County has not increased, and perhaps even decreased, despite the increase in prevalence of CA-MRSA over the years. A similar decrease in the incidence of SSTIs (though not specific to NLEC) was described recently at a Chicago medical center. ${ }^{18}$

Similar to the 1999 study, our data showed a significant increase in incidence of NLEC with increasing age. ${ }^{7}$ Unlike the 1999 study, however, NLEC incidence differed by sex.

According to age-adjusted rates, the incidence of NLEC among males was similar between 1999 and 2013 (227.1 and 225.8 per 100,000, respectively; $\mathrm{P}=.82$ ), whereas the incidence among females was significantly lower in 2013 (181.2 and 132.6 per 100,000; $\mathrm{P}=.009$ ). The present finding of a lower rate among females compared to males is consistent with the comparisons of sex-specific incidence between 1999 and 2013, which suggest that the current rate among females is lower than previously reported.

While we did not have prevalence rates for CA-MRSA specific to Olmsted County, the rates described for Minnesota can be considered representative, as the sentinel hospitals were chosen to represent demographics of patients served across the state. ${ }^{12,19}$ A decrease in incidence of NLEC despite prevalence of CA-MRSA is noteworthy because providers often use antimicrobials that include coverage for CA-MRSA for NLEC. ${ }^{20}$ In our cohort, $63 \%$ of patients received cephalexin; however $16 \%$ and $11 \%$ of patients received trimethoprim/ sulfamethoxazole or vancomycin, respectively (data not shown), despite absence of purulence. Current IDSA guidelines acknowledge that "MRSA is an unusual cause of typical cellulitis"; in fact, they suggest that it is inappropriate to designate the term "cellulitis" to any SSTI that includes a collection of purulence. ${ }^{2}$ Nevertheless, current guidelines suggest clindamycin (which covers MRSA) as an option for treatment of mild/ moderate non-purulent SSTIs. ${ }^{2}$ Clindamycin as monotherapy for NLEC may be problematic given the increased risk of diarrhea, ${ }^{21}$ including fatal and nonfatal adverse events relating to Clostridium difficile infections. ${ }^{22}$

Our data demonstrated that an increased incidence of SSTIs mediated by a rising prevalence of CA-MRSA has had little impact on the incidence of NLEC, suggesting that empiric coverage of CA-MRSA may be unnecessary. In a randomized controlled trial comparing effectiveness of trimethoprim/sufamethoxazole vs. cephalexin for treatment of uncomplicated (non-purulent) cellulitis, combination therapy with both agents did not impact outcomes of cellulitis compared to cephalexin monotherapy. ${ }^{23}$ Similarly, another randomized controlled trial comparing outcomes, adding clindamycin to flucoxacillin did not improve outcomes of cellulitis compared to flucoxacillin monotherapy. ${ }^{21} \mathrm{An}$ analysis of outcomes of empiric therapy in the present study would have strengthened our conclusions if this showed that patients treated empirically without CA-MRSA coverage did no worse than those treated with CA-MRSA coverage. However, the retrospective nature of our study made it difficult to obtain these data because many patients did not return for evaluation after 
initial treatment; while one may assume that they improved, this assumption cannot be verified. Our data ultimately can be used, with data from other studies, to impact the designation of empiric antibiotic regimens promulgated by future guidelines to enhance clinical outcomes and promote antimicrobial stewardship in the initial management of NLEC.

Finally, the impact of seasonality on SSTIs occurrence has been described with more cases in the summer or wetter months. ${ }^{7,} 18$ Review of available literature identified only four studies specifically describing seasonal variations in NLEC. ${ }^{7,24-26}$ Moreover, the only US study to note seasonal variation in NLEC was the one conducted in our population in 1999. ${ }^{7}$ Recognition of seasonality in the epidemiology of NLEC is important as we consider educational aspects for both clinicians and patients. This could include an enhanced recognition by clinicians of the diagnosis of NLEC in the differential diagnosis of this syndrome among a wide variety of other diseases that can mimic cellulitis. ${ }^{27}$ In addition, patient education for those at high risk of an initial bout of cellulitis or a recurrent bout could include aggressive treatment of tinea pedis for those with this infection and the use of protective clothing during warmer months.

\section{Limitations}

The retrospective design relies on accurate medical records with proper documentation and diagnostic coding for capture of true cases, and assumes patients with NLEC will seek healthcare. This could introduce bias toward more severe cases; however, this is unlikely as the large majority of patients did not require hospitalization for NLEC. Additionally, medical records were individually examined to verify strict adherence to a standardized case definition.

\section{Conclusion}

The incidence of NLEC in Olmsted County was lower in 2013 than that reported in 1999 prior to the emergence of CA-MRSA. This suggests that, unlike skin abscess, CA-MRSA does not play a significant role as a cause of NLEC. This, coupled with the results of a clinical trial that examined the role of CA-MRSA coverage in non-purulent cellulitis, suggests that empiric coverage of CA-MRSA in cases of NLEC is unnecessary. In addition, cases of NLEC are seasonally distributed, with more cases occurring in spring/summer. Revised clinical recommendations for empiric treatment and risk factor modification to prevent NLEC should therefore be considered.

\section{Supplementary Material}

Refer to Web version on PubMed Central for supplementary material.

\section{Acknowledgments}

Author Contributions: JRM led project design and execution, secondary chart review, data abstraction, simple statistical analysis, literature review, initial drafting, and subsequent manuscript revisions. DWC and EMT participated in literature review, secondary data abstraction, initial drafting and subsequent manuscript revision. BDL performed major statistical analysis and manuscript revision. LMB participated in project design, tertiary 
chart review, and manuscript revisions. All authors participated in critical review and approval of final version of manuscript.

Non-author Contributions: We would like to thank Donna Lawson CCRP and her team of nurse abstractors for their hard work and dedication to complete the initial data abstraction of all potential case patients in the REP database.

Conflicts of interest: JRM, DWC, EMT, BDL, LMB - No conflicts of interest for any authors.

Funding: This publication was supported by the Mayo Foundation for Research and Education through the Division of Infectious Diseases, by the Rochester Epidemiology Project (Grant number R01-AG034676; Principal Investigators: Walter A. Rocca, MD, and Barbara P. Yawn, MD, MSc), and by Grant Number UL1 TR000135 from the National Center for Advancing Translational Sciences (NCATS). Its contents are solely the responsibility of the authors and do not necessarily represent the official views of the NIH. No other sources of funding were used.

\section{Abbreviations}

CA-MRSA Community-acquired methicillin-resistant Staphylococcus aureus

NLEC Non-purulent lower extremity cellulitis

SSTI Skin/soft tissue infection

REP Rochester Epidemiology Project

\section{References}

1. Swartz MN. Clinical practice. Cellulitis. The New England journal of medicine. 2004; 350(9):904912. [PubMed: 14985488]

2. Stevens DL, Bisno AL, Chambers HF, et al. Practice guidelines for the diagnosis and management of skin and soft tissue infections: 2014 update by the Infectious Diseases Society of America. Clin Infect Dis. 2014; 59(2):e10-52. [PubMed: 24973422]

3. AM, DF, JC. London EOoPCaS. Morbidity Statistics From General Practice: Fourth National Study. Vol. 188. London, England: 1992.

4. Ellis Simonsen SM, van Orman ER, Hatch BE, et al. Cellulitis incidence in a defined population. Epidemiology \& Infection. 2006; 134(2):293-299. [PubMed: 16490133]

5. GRL. Cellulitis Among Active Duty Servicemembers, US Armed Forces, 1998-2001. Med Surveill Monthly Rep (MSMR). 2002; 8(7):6-9.

6. McCormic ZD, Balihe MN, Havas KA. Bacterial skin infections, active component, U.S. Armed Forces, 2000-2012. Med Surveill Monthly Rep (MSMR). 2013; 20(12):2-7.

7. McNamara DR, Tleyjeh IM, Berbari EF, et al. Incidence of lower-extremity cellulitis: a populationbased study in Olmsted county, Minnesota. Mayo Clin Proc. 2007; 82(7):817-821. [PubMed: 17605961]

8. Levine DP, Cushing RD, Jui J, Brown WJ. Community-acquired methicillin-resistant Staphylococcus aureus endocarditis in the Detroit Medical Center. Ann Intern Med. 1982; 97(3): 330-338. [PubMed: 7114630]

9. Centers for Disease C, Prevention. Four pediatric deaths from community-acquired methicillinresistant Staphylococcus aureus - Minnesota and North Dakota, 1997-1999. MMWR - Morbidity \& Mortality Weekly Report. 1999; 48(32):707-710. [PubMed: 21033181]

10. Qualls ML, Mooney MM, Camargo CA Jr, Zucconi T, Hooper DC, Pallin DJ. Emergency department visit rates for abscess versus other skin infections during the emergence of communityassociated methicillin-resistant Staphylococcus aureus, 1997-2007. Clinical infectious diseases : an official publication of the Infectious Diseases Society of America. 2012; 55(1):103-105. [PubMed: 22460965]

11. Fridkin SK, Hageman JC, Morrison M, et al. Methicillin-Resistant Staphylococcus aureus Disease in Three Communities. New England Journal of Medicine. 2005; 352(14):1436-1444. [PubMed: 15814879] 
12. Como-Sabetti K, Harriman KH, Buck JM, Glennen A, Boxrud DJ, Lynfield R. CommunityAssociated Methicillin-Resistant Staphylococcus aureus: Trends in Case and Isolate Characteristics from Six Years of Prospective Surveillance. Public Health Reports. 2009; 124(3): 427-435. [PubMed: 19445419]

13. Rocca WA, Yawn BP, St Sauver JL, Grossardt BR, Melton LJ 3rd. History of the Rochester Epidemiology Project: half a century of medical records linkage in a US population. Mayo Clin Proc. 2012; 87(12):1202-1213. [PubMed: 23199802]

14. Bjornsdottir S, Gottfredsson M, Thorisdottir AS, et al. Risk factors for acute cellulitis of the lower limb: a prospective case-control study. Clin Infect Dis. 2005; 41(10):1416-1422. [PubMed: 16231251]

15. Thomas KS, Crook AM, Nunn AJ, et al. Penicillin to prevent recurrent leg cellulitis. The New England journal of medicine. 2013; 368(18):1695-1703. [PubMed: 23635049]

16. St Sauver JL, Grossardt BR, Yawn BP, Melton LJ 3rd, Rocca WA. Use of a medical records linkage system to enumerate a dynamic population over time: the Rochester epidemiology project. Am J Epidemiol. 2011; 173(9):1059-1068. [PubMed: 21430193]

17. United States Department of Commerce. [Accessed March 30, 2017] United States Census Bureau website. www.census.gov

18. Morgan E, Daum RS, David MZ. Decreasing Incidence of Skin and Soft Tissue Infections With a Seasonal Pattern at an Academic Medical Center, 2006-2014. Open Forum Infectious Diseases. 2016; 3(4)

19. Naimi TS, LeDell KH, Como-Sabetti K, et al. Comparison of community- and health careassociated methicillin-resistant staphylococcus aureus infection. JAMA. 2003; 290(22):29762984. [PubMed: 14665659]

20. Meddles-Torres $\mathrm{C}, \mathrm{Hu} \mathrm{S}$, Jurgens C. Changes in prescriptive practices in skin and soft tissue infections associated with the increased occurrence of community acquired methicillin resistant Staphylococcus aureus. J Infect Public Health. 2013; 6(6):423-430. [PubMed: 23999333]

21. Brindle R, Williams OM, Davies P, et al. Adjunctive clindamycin for cellulitis: a clinical trial comparing flucloxacillin with or without clindamycin for the treatment of limb cellulitis. BMJ Open. 2017; 7(3):e013260.

22. Thornhill MH, Dayer MJ, Prendergast B, Baddour LM, Jones S, Lockhart PB. Incidence and nature of adverse reactions to antibiotics used as endocarditis prophylaxis. J Antimicrob Chemother. 2015; 70(8):2382-2388. [PubMed: 25925595]

23. Pallin DJ, Binder WD, Allen MB, et al. Clinical trial: comparative effectiveness of cephalexin plus trimethoprim-sulfamethoxazole versus cephalexin alone for treatment of uncomplicated cellulitis: a randomized controlled trial. Clin Infect Dis. 2013; 56(12):1754-1762. [PubMed: 23457080]

24. Haydock SF, Bornshin S, Wall EC, Connick RM. Admissions to a U.K. teaching hospital with nonnecrotizing lower limb cellulitis show a marked seasonal variation. The British journal of dermatology. 2007; 157(5):1047-1048. [PubMed: 17711519]

25. Macario-Barrel A, Zeghnoun A, Young P, et al. Influence of environmental temperature on the occurrence of non-necrotizing cellulitis of the leg. The British journal of dermatology. 2004; 150(1):155-156. [PubMed: 14746635]

26. Szyszkowicz M, Porada E, Kaplan GG, Rowe BH. Ambient ozone and emergency department visits for cellulitis. International journal of environmental research and public health. 2010; 7(11): 4078-4088. [PubMed: 21139878]

27. Hirschmann JV, Raugi GJ. Lower limb cellulitis and its mimics: part II. Conditions that simulate lower limb cellulitis. J Am Acad Dermatol. 2012; 67(2):177, e171-179. quiz 185-176. [PubMed: 22794816] 


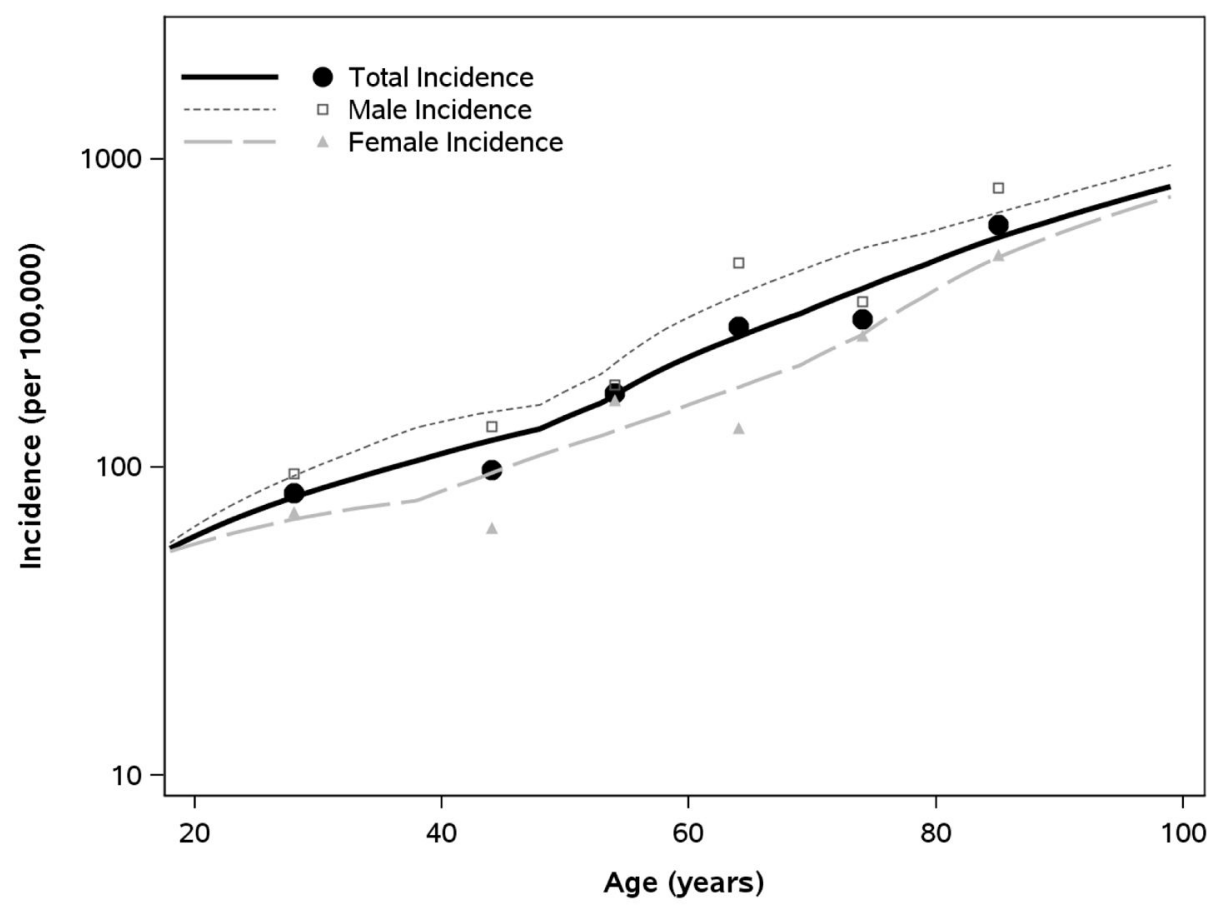

Figure 1.

Relationship of Age and Sex with Incidence of Non-purulent Lower Extremity Cellulitis in Olmsted County (2013)

Note: Incidence data specifying counts of NLEC cases (numerator) and counts of total atrisk adults (denominator) were organized in strata specific to sex and each single year of age (18-100 years). Using this granular form of data, smoothing techniques (loess algorithm) were used to estimate incidence in relation to age and sex. Symbols represent sex-specific incidence rates estimated according to age groups (those shown in Table 2) to provide a crude verification of the smoothed trends. In a multivariable Poisson regression model, rates of incidence were higher in males and increased with age for both sexes $(\mathrm{P}<.001$ for both age and sex effects). 
Table 1

Baseline Characteristics of Incident Cases of Confirmed Non-purulent Lower Extremity Cellulitis ( $\mathrm{n}=195)$

\begin{tabular}{|c|c|c|}
\hline Characteristic & $N$ & No. $(\%)$ or Median (IQR) \\
\hline Age & 195 & $61.5(45.0,73.1)$ \\
\hline Male Sex & 195 & $113(57.9 \%)$ \\
\hline Caucasian Race & 195 & $188(96.4 \%)$ \\
\hline Basic Metabolic Index & 176 & $32.6(26.0,38.1)$ \\
\hline \multicolumn{3}{|l|}{ Presenting Signs/Symptoms } \\
\hline Erythema & 195 & $195(100.0 \%)$ \\
\hline Warmth & 195 & $142(72.8 \%)$ \\
\hline Tenderness & 195 & $100(51.3 \%)$ \\
\hline Edema & 195 & $161(82.6 \%)$ \\
\hline Pain & 195 & $139(71.3 \%)$ \\
\hline Presenting Anatomical Site & 195 & \\
\hline Toe & & $23(11.8 \%)$ \\
\hline Foot & & $34(17.4 \%)$ \\
\hline Ankle & & $12(6.2 \%)$ \\
\hline Leg & & $50(25.6 \%)$ \\
\hline Knee & & $10(5.1 \%)$ \\
\hline Thigh & & $8(4.1 \%)$ \\
\hline Multiple & & $19(9.7 \%)$ \\
\hline LE Not Otherwise Specified $^{a}$ & & $39(20.0 \%)$ \\
\hline Affected Side & 195 & \\
\hline Left & & $92(47.2 \%)$ \\
\hline Right & & $96(49.2 \%)$ \\
\hline Both & & $7(3.6 \%)$ \\
\hline Diagnosis Location & 195 & \\
\hline Inpatient & & $4(2.1 \%)$ \\
\hline Outpatient & & $131(67.2 \%)$ \\
\hline Emergency Department & & $60(30.8 \%)$ \\
\hline Duration of Symptoms (days) $b$ & 162 & $2.8(1.0,5.0)$ \\
\hline Temperature at Diagnosis & 148 & $36.7(36.4,37.0)$ \\
\hline Fever Documented $^{c}$ & 148 & $11(7.4 \%)$ \\
\hline Required Hospitalization & 195 & $28(14.4 \%)$ \\
\hline Length of Hospitalization (days) & 28 & $3.5(2.0,6.0)$ \\
\hline Antibiotics Prescribed & 195 & $195(100.0 \%)$ \\
\hline Duration of Antibiotics (days) & 195 & $10(9,10)$ \\
\hline Previous History of Cellulitis & 158 & $45(28.5 \%)$ \\
\hline
\end{tabular}

Mayo Clin Proc. Author manuscript; available in PMC 2018 August 01. 
$c_{\text {Prior to seeking medical care; }}$

${ }^{d}$ At initial presentation 
Table 2

Incidence of Non-purulent Lower Extremity Cellulitis (NLEC) in Olmsted County, Minnesota (2013) by Age, Sex, and Seasonal Periods

\begin{tabular}{|c|c|c|c|}
\hline & Females & Males & Both Sexes \\
\hline \multicolumn{4}{|c|}{ Unadjusted Incidence } \\
\hline Age & \multicolumn{3}{|c|}{ Number of cases (incidence rate, per 100,000 persons) } \\
\hline $18-39$ & $18(71.3)$ & $21(95.3)$ & $39(82.5)$ \\
\hline $40-49$ & $6(63.1)$ & $12(135.0)$ & $18(97.8)$ \\
\hline $50-59$ & $19(164.5)$ & $19(184.2)$ & $38(173.8)$ \\
\hline $60-69$ & $10(133.3)$ & $30(458.4)$ & $40(284.7)$ \\
\hline $70-79$ & $12(265.5)$ & $13(343.2)$ & $25(300.9)$ \\
\hline $80-99$ & $17(487.4)$ & $18(802.1)$ & $35(610.6)$ \\
\hline All Ages & $82(132.6)$ & $113(210.0)$ & $195(168.6)$ \\
\hline \multicolumn{4}{|l|}{ Adjusted Incidence } \\
\hline Season & \multicolumn{3}{|c|}{ Incidence rate $(95 \% \mathrm{CI})$, per 100,000 persons } \\
\hline Warm Months ${ }^{\dagger}$ & $193.8(139.5,248.1)$ & $260.3(189.9,330.7)$ & $224.6(180.9,268.4)$ \\
\hline Cold Months ${ }^{* t}$ & $90.1(58.5,121.7)$ & $201.1(149.0,253.1)$ & $142.3(112.8,171.9)$ \\
\hline Overall & $133.3(104.1,162.5)$ & $225.8(183.5,268.0)$ & $176.6(151.5,201.7)$ \\
\hline
\end{tabular}

Note: Unadjusted incidence is presented as number of NLEC cases along with the crude incidence rate for all age-sex groups, while adjusted incidence rates computed by season or overall are accompanied with $95 \%$ confidence intervals and are age- and sex-standardized (or agestandardized for sex-specific incidence) to the US white population in 2010. Multivariable Poisson regression analysis revealed independent effects of age, sex and season on rates of incidence, with older age $(\mathrm{P}<.001)$, male sex $(\mathrm{P}<.001)$ and "warm" season $(\mathrm{P}=.001)$ each associated with higher incidence of NLEC.

${ }^{\dagger}$ Pre-specified interval of May-September was used to represent warm seasonal months

${ }^{t}$ Remaining months of January-April and October-December were considered to be cold seasonal months 